BMJ Open

Sport \&

Exercise

Medicine

\title{
Is ultrasound-guided lavage an effective intervention for rotator cuff calcific tendinopathy? A systematic review with a meta-analysis of randomised controlled trials
}

Simon Lafrance, ${ }^{\oplus 1}$ Patrick Doiron-Cadrin, ${ }^{1}$ Marie Saulnier, ${ }^{1}$ Martin Lamontagne, ${ }^{2,3}$ Nathalie J Bureau, ${ }^{3,4}$ Joseph-Omer Dyer, ${ }^{5}$ Jean-Sébastien Roy, ${ }^{6,7}$

François Desmeules ${ }^{1,5}$

To cite: Lafrance $S$, Doiron-Cadrin P, Saulnier M, et al. Is ultrasoundguided lavage an effective intervention for rotator cuff calcific tendinopathy? A systematic review with a meta-analysis of randomised controlled trials. BMJ Open Sport \& Exercise Medicine 2019;5:e000506. doi:10.1136/ bmjsem-2018-000506

- Additional material is published online only. To view please visit the journal online (http://dx.doi.org/10.1136/ bmjsem-2018-000506).

Accepted 21 February 2019

\section{Check for updates}

\section{(C) Author(s) (or their} employer(s)) 2019. Re-use permitted under CC BY-NC. No commercial re-use. See rights and permissions. Published by BMJ.

For numbered affiliations see end of article.

\section{Correspondence to} Dr François Desmeules; f.desmeules@umontreal.ca

\section{ABSTRACT}

Background Rotator cuff calcific tendinopathy is characterised by the deposition of hydroxyapatite crystals in one of the rotator cuff tendons and can be managed by ultrasound-guided lavage. However, evidence regarding the efficacy of ultrasound-guided lavage for rotator cuff calcific tendinopathy remains inconclusive. The aim of this systematic review and meta-analysis is to update the available evidence on the efficacy of ultrasound-guided lavage in adults with rotator cuff calcific tendinopathy. Methods A literature search was conducted up to April 2018 in four bibliographic databases to identify randomised control trials that compared ultrasound-guided lavage alone with other interventions to treat rotator cuff calcific tendinopathy. Randomised control trials were assessed with the Cochrane Risk of Bias Tool. Meta-analyses and/or qualitative synthesis of the evidence were performed.

Results Three randomised control trials were included. Pooled results for pain $(n=226)$ indicated that ultrasoundguided lavage may significantly decrease pain when compared with shockwave therapy, with a mean difference of -1.98 out of 10 points $(95 \% \mathrm{Cl}-2.52$ to -1.45$)$ in the short term and of $-1.84(95 \% \mathrm{Cl}-2.63$ to -1.04$)$ in the long term. Based on one trial $(n=25)$, ultrasound-guided lavage significantly improved function when compared with shockwave therapy $(p<0.05)$. Based on another trial $(n=48)$, the addition of ultrasound-guided lavage to a corticosteroid injection significantly improves function in the long term $(p<0.05)$.

Conclusion For individuals with rotator cuff calcific tendinopathy, low-quality evidence suggests that ultrasound-guided lavage is more effective than shockwave therapy or a corticosteroid injection alone. Future trials could modify the present conclusions.

\section{TRIAL REGISTRATION NUMBER} CRD42018095858.

\section{INTRODUCTION}

Shoulder pain is highly prevalent, and among musculoskeletal disorders, it is the third most common reason for visiting a primary care

\section{What is already known?}

Rotator cuff calcific tendinopathy is characterised by the deposition of hydroxyapatite crystals in one of the rotator cuff tendons.

- Rotator cuff calcific deposit is more prevalent in adults with subacromial pain syndrome.

- It is unclear whether ultrasound-guided lavage has beneficial effects compared with conventional interventions in the treatment of rotator cuff calcific tendinopathy.

\section{What are the new findings?}

- Low-quality evidence suggests that ultrasound-guided lavage with a corticosteroid injection is more effective than shockwave therapy in the treatment of rotator cuff calcific tendinopathy.

- Low-quality evidence suggests that the addition of ultrasound-guided lavage to a corticosteroid injection is beneficial for adults with rotator cuff calcific tendinopathy.

physician. ${ }^{1-3}$ One common shoulder disorder resulting in pain and loss of function is rotator cuff (RC) calcific tendinopathy. The estimated prevalence of $\mathrm{RC}$ calcific deposit is of $7.8 \%$ in asymptomatic adults and of $42.5 \%$ in adults with subacromial pain syndrome. ${ }^{4}$ The prevalence is higher in middle-age adults and in women. ${ }^{4-6}$ RC calcific tendinopathy is characterised by the deposition of hydroxyapatite crystals in one of the RC tendons, ${ }^{7}$ most commonly the supraspinatus and infraspinatus tendons. ${ }^{489} \mathrm{RC}$ calcific tendinopathy is often classified into three stages which are precalcific, calcific and postcalcific stages $^{810}$ and the time course of these different stages can evolve within a few months to several years. ${ }^{10}$ 
Initial conservative treatment of RG calcific tendinopathy consists of physiotherapy including an exercise programme $^{11}$ and may also include therapeutic ultrasound. ${ }^{12}$ Oral non-steroidal anti-inflammatory drugs can also be prescribed for pain management. ${ }^{10}$ If initial conservative treatment fails, other treatments may be prescribed, as a second-line treatment, such as corticosteroid injections or shockwave therapy. ${ }^{810}$ Arthroscopic debridement may be considered only in severe chronic recalcitrant cases. ${ }^{8} 10$

Ultrasound-guided lavage (US-guided lavage), as a second-line treatment, has gained popularity in the last decades. US-guided lavage is a minimally invasive intervention consisting in the introduction of a needle into the calcific deposit. A saline and/or an anaesthetic solution is then injected into the calcification with several short injections, each followed by release of pressure on the plunger to allow the solution and calcific material to evacuate back into the syringe. ${ }^{8}$ US-guided lavage is often followed by a subacromial corticosteroid injection. ${ }^{8}$ Although several studies have investigated the use of US-guided lavage, it is unclear whether this intervention has beneficial effects compared with conventional interventions. There is a lack of standardisation in the definition of the US-guided lavage intervention. ${ }^{13}$ Most reviews on US-guided lavage also included needle fragmentation without lavage. ${ }^{6} 101415$ US-guided needle fragmentation is a procedure that is similar to US-guided lavage; however, it does not include the lavage procedure and may not be as efficient for calcification removal. ${ }^{813}$ Pooling of US-guided lavage and needle fragmentation without lavage is then debatable since those two interventions are different. ${ }^{13}$

Louwerens $e t a l^{6}$ published a systematic review on the efficacy of interventions for chronic symptomatic RC calcific tendinopathy. However, they included only one randomised control trial (RCT) on US-guided lavage. ${ }^{16}$ They concluded that US-guided lavage with a corticosteroid injection was no more effective than a corticosteroid injection alone in terms of function. Two other network meta-analyses have been published more recently in relation to treatments of RC calcific tendinopathy ${ }^{10}{ }^{14}$ Both reviews recommended the use of US-guided lavage for RC calcific tendinopathy. However, US-guided lavage and US-guided needling fragmentation were pooled together. Moreover, none of these reviews analysed the efficacy of US-guided lavage on self-reported function and the authors did not include all relevant RCTs on this topic. Consequently, an update of the available evidence is warranted.

The aim of this systematic review and meta-analysis was to update the available evidence on the efficacy of US-guided lavage compared with any other interventions in adults with RC calcific tendinopathy.

\section{METHODS}

The study protocol is available online on PROSPERO (https://www.crd.york.ac.uk/prospero/).

\section{Literature search}

An electronic bibliographical search of four databases (Medline, Embase, Cochrane Central and CINAHL) was performed up to April 2018 using terms related to shoulder, RC, tendinopathy, calcified, needling and irrigation (see online supplemental material for full strategy). The reference lists of all identified published studies and previous systematic reviews were checked for any additional eligible trials.

\section{Study selection}

Two reviewers (SL and MS) independently reviewed titles and abstracts to identify trials of interest. Consensus of the two reviewers was needed to include the studies. A third reviewer (PD-C) was available if a consensus was not achieved by the two initial reviewers.

Articles were included if they met the following inclusion criteria: (1) participants were adults diagnosed with RC calcific tendinopathy confirmed by radiography or ultrasound imaging; (2) the intervention included US-guided lavage (specifically lavage and not only fragmentation) alone or in conjunction with another intervention such as a corticosteroid injection; (3) the intervention was compared with a placebo or any other intervention; (4) at least one outcome measure was related to pain, function, health-related quality of life, return to work, satisfaction, a global rating of change or to calcification size; (5) the study was an RCT and (6) was published either in English or in French. Studies were excluded if participants presented a full-thickness RC tear, a postsurgical condition or any other concomitant shoulder, upper limb or neck disorder.

\section{Data extraction}

Data of included studies were extracted with a standardised form that documented the number of participants, their characteristics, the type of intervention (US-guided lavage alone or in conjunction with another intervention), the control intervention, outcome measures, length of the follow-up and results (table 1). When data were missing, or incomplete, original authors were contacted.

\section{Risk of bias appraisal tool}

The methodological quality of the included RCTs was assessed using the Cochrane Risk of Bias tool ${ }^{17}$ by two independent evaluators (SL and MS). The final score was obtained through consensus. In case of disagreement, a third reviewer (PD-C) was available to achieve a consensus. For each trial, a total final score was also calculated and transformed in percentage allowing a summary measure of the overall risk of bias.

\section{Data synthesis}

Results from studies with similar comparators and outcome measures such as pain, function, quality of life and measures of impairment such range of motion, shoulder strength or size of the calcific deposit were considered for pooling into separate meta-analyses. Pooled mean differences were calculated. Pooled 


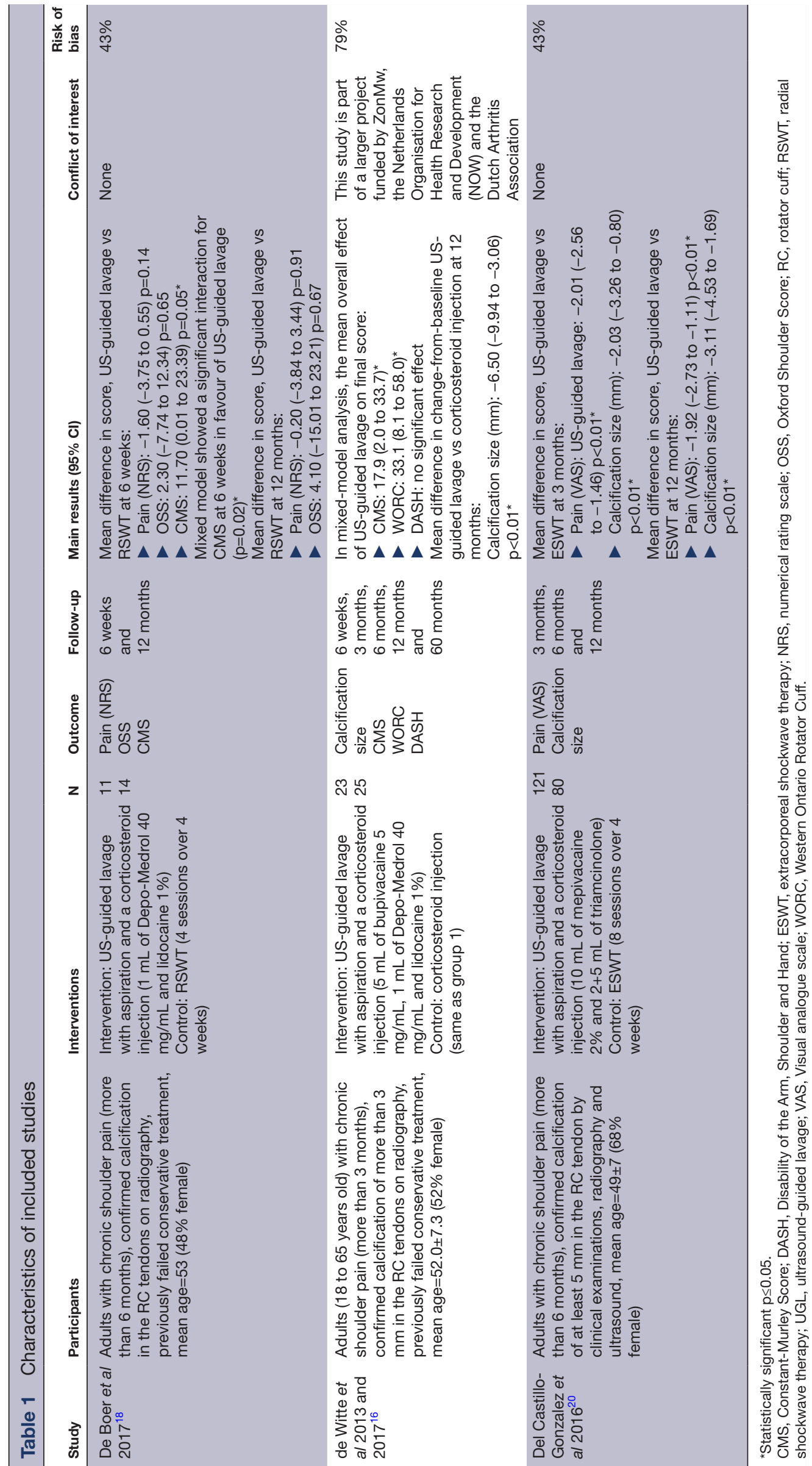




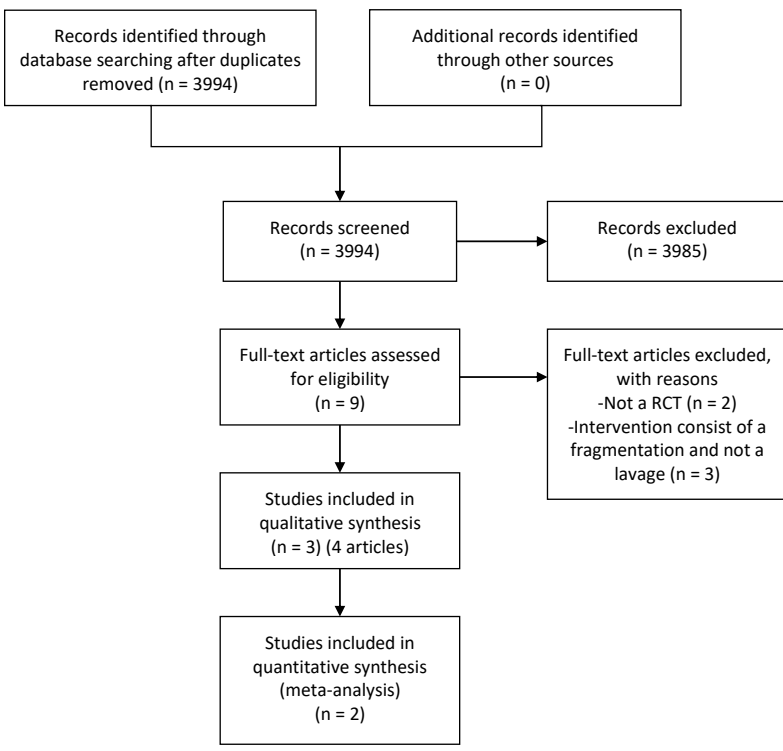

Figure 1 Schematic breakdown of literature search results. $\mathrm{RCT}$, randomised controlled trial.

estimates with $95 \%$ CIs were calculated using Review Manager (RevMan V.5.3; The Cochrane Collaboration, Copenhagen, Denmark). Alpha level was set at 0.05. The inverse variance method was used to weigh each study. Analyses were performed with fixed effects. Visual inspection of the forest plots was performed. Only meta-analyses without a significant degree of heterogeneity were kept and reported $\left(\chi^{2} \mathrm{p}>0.10\right.$ and $\left.\mathrm{I}^{2}<60 \%\right)$. When SDs from original studies were missing, estimations were made using Review Manager and according to the Cochrane Collaboration recommendations on how to handle missing data. ${ }^{17}$ For studies not included in the meta-analyses, a qualitative analysis was performed.

\section{RESULTS}

From the nine potentially relevant articles identified through titles and abstract review, three RCTs met the eligibility criteria after full-text review, ${ }^{16}$ 18-20 one including the original publication and a 5-year

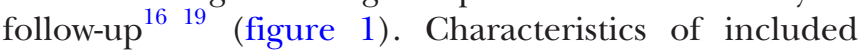
studies are presented in table 1.

In every RCT, the population was composed of adults with chronic RC calcific tendinopathy of at least $3^{16} 19$ to 6 months' duration. ${ }^{18}{ }^{20} \mathrm{RC}$ calcific tendinopathy was confirmed by radiography and/or ultrasound. The mean age of participants ranged from 49 to 53 years. In two trials, the population was strictly composed of participants who previously failed a conservative treatment. ${ }^{16} 1819$

\section{Interventions and comparators}

One RCT compared US-guided lavage with aspiration and a corticosteroid injection to a corticosteroid injection alone. $^{1619}$ Two RCTs compared US-guided lavage with aspiration followed by a corticosteroid injection to shockwave therapy. De Boer et $a l^{18}$ used radial shockwave therapy for four sessions while Del Castillo-Gonzalez et $a t^{20}$ used extracorporeal shockwave therapy (ESWT) for eight sessions.

\section{Outcome measures}

For pain assessment, one RCT used a visual analogue scale $^{20}$ and one used the numerical rating scale. ${ }^{18}$ However, the method to assess pain was not further described in either studies. For self-reported function, the study by De Boer et $a l^{18}$ used the Oxford Shoulder Score while the study by de Witte et $a l^{1619}$ used the Western Ontario Rotator Cuff questionnaire (WORC) and the Disability of the Shoulder, Arm and Hand questionnaire (DASH). These two RCTs also reported the Constant-Murley Score (CMS) which is a composite functional measure with sections on pain, function, strength and ROM. Two RCTs also monitored the reduction in the calcification size (mm). ${ }^{16} 1920$

\section{Risk-of-bias appraisal}

The Cochrane risk-of-bias tool mean score across all included RCTs was $55 \% \pm 17 \%$. One study was considered at low risk of bias with a total score of $79 \%,{ }^{16}$ and the two other studies were considered at high risk of bias with total scores of $43 \% .^{18}{ }^{20}$ Blinding of the personnel was not possible due to the nature of the intervention while participants and assessor blinding was achieved in only one RCT. ${ }^{1619}$ As other sources of bias, a noticeable discrepancy between baseline scores across the intervention and the control groups was observed in the RCT published by de Witte et al. ${ }^{1619}$ However, their analyses were conducted while adjusting for this potential confusion bias. In the trial published by De Boer et $a l,{ }^{18}$ an important proportion of participants $(36 \%)$ in the shockwave group did not complete the study due to adverse events (figures 2 and 3 ).

\section{Efficacy of US-guided lavage with a corticosteroid injection compared to shockwave therapy}

\section{Pain-related outcomes}

Two high risk of bias RCTs assessed the efficacy of US-guided lavage with a corticosteroid injection compared with shockwave therapy on pain. ${ }^{18} 20$ The two RCTs were pooled together into two meta-analyses (figure 4). The mean differences between groups showed a significant effect in favour of US-guided lavage with a corticosteroid injection for pain reduction in the short term (at 6 weeks or 3 months, $\mathrm{p}<0.01$ ) and at 12 months $(\mathrm{p}<0.01)$. The mean difference was -1.84 out 10 points (95\% CI -2.52 to -1.45$)$ for short-term effects and -1.96 out of 10 points $(95 \%$ CI -2.63 to -1.04$)$ at 12 months. The magnitude of these differences is above the minimal clinically important difference (MCID) of 1.4 for shoulder pain. ${ }^{21}$ 


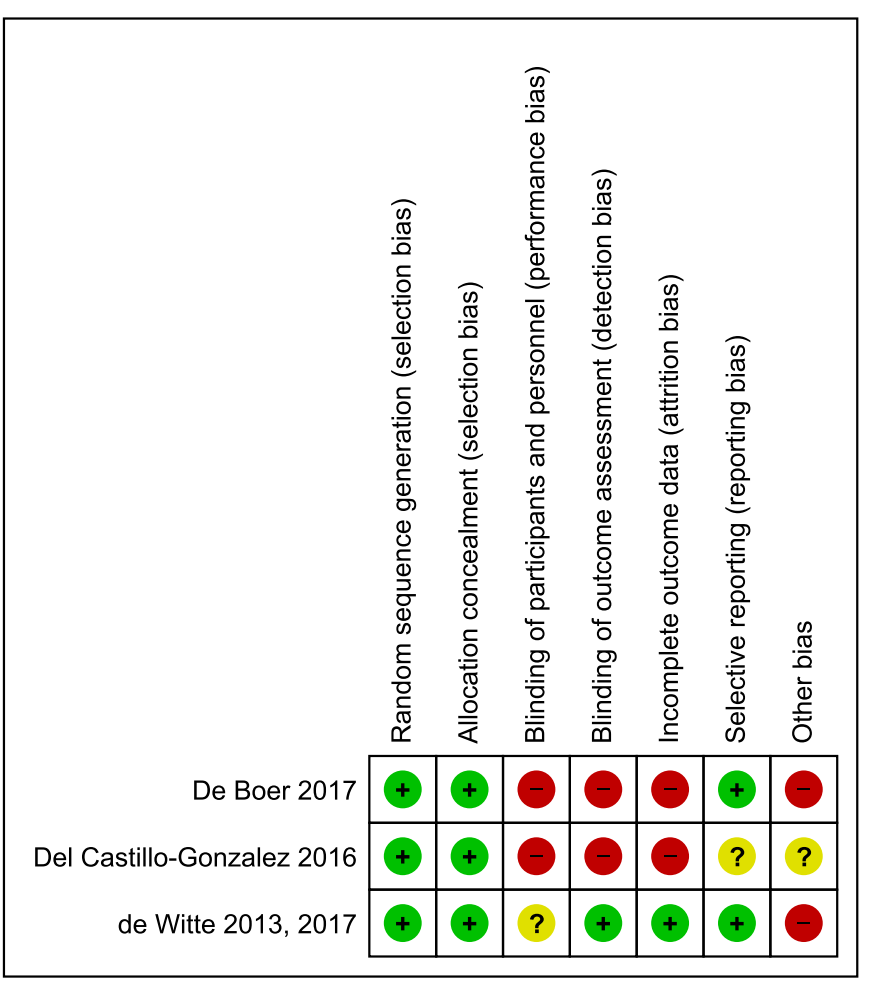

Figure 2 Detailed methodological assessment of included studies using the Cochrane risk-of-bias tool. Green: low risk of bias, red: high risk of bias, yellow: unclear or unknown risk of bias.

Functional related outcomes

The high risk of bias RCT by De Boer et al ${ }^{18}$ assessed the efficacy of US-guided lavage with a corticosteroid injection compared with shockwave therapy (RSWT) on self-reported function. The Oxford Shoulder Score improved in both groups at 6 weeks and 12 months; however, no significant statistical differences between groups were observed ( $\mathrm{p} \geq 0.05)$. For the CMS, the mean difference in final score was significantly higher for the US-guided lavage group by 11.7 out of 100 points $(95 \%$ CI 0.01 to $23.39, \mathrm{p}<0.05$ ) at 6 weeks. Their analysis also showed a significant statistical difference between groups in the CMS in favour of the US-guided lavage at 6 weeks $(p=0.02)$. The magnitude of the differences observed is within the range of previously reported MCID for the CMS which varies between $10.4^{22}$ and 18 points. ${ }^{23}$

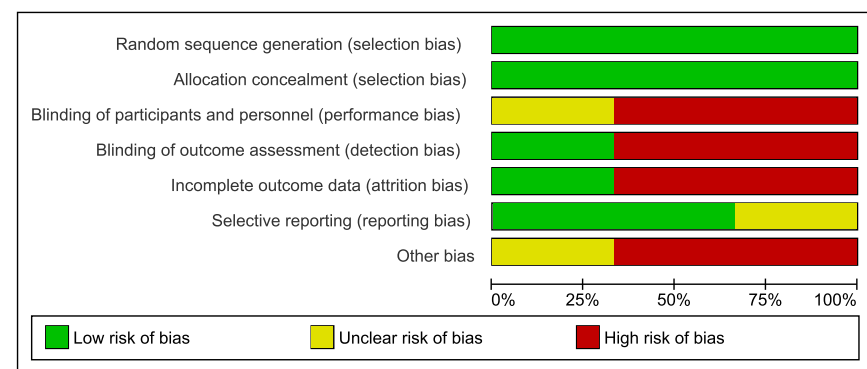

Figure 3 Risk-of-bias graph: review authors' judgements about each risk-of-bias item, presented as percentages across all included studies.
Calcification size

The high risk of bias RCT by Del Castillo-Gonzalez et $a l^{20}$ also assessed the efficacy of US-guided lavage with a corticosteroid injection compared with shockwave therapy (ESWT) on calcification size reduction. Both interventions lead to statistically significant reductions in the calcification size over time $(p<0.01)$. However, the mean differences between groups showed a significant effect in favour of US-guided lavage with a corticosteroid injection compared with shockwave therapy to decrease calcification size at 3, 6 and 12 months $(\mathrm{p}<0.01)$.

\section{Efficacy of US-guided lavage with a corticosteroid injection compared with a corticosteroid injection alone \\ Functional related outcome}

The low risk of bias RCT by de Witte et $a l^{16}{ }^{19}$ assessed the efficacy of US-guided lavage combined with a corticosteroid injection compared with a corticosteroid injection alone on functional related outcome. At 12 months, both groups improved. The mean overall effect of US-guided lavage compared with a corticosteroid injection for the CMS at 12 months was 17.9 out of 100 points (95\% CI 2.0 to 33.7) while the effect on the WORC score at 12 months was 33.1 out of 100 points (95\% CI 8.1 to 58.0). There was no significant difference between groups on the DASH score $(\mathrm{p} \geq 0.05)$. The magnitude of the differences observed for the CMS is within the range of previously reported MCID which varies between $10.4^{22}$ and 18 points. ${ }^{23}$ For the WORC, the magnitude of the difference is above previously reported MCID which varies between $13.1^{24}$ to 14.3 points. $^{25}$

\section{Calcification size}

De Witte $e t a l^{1619}$ also assessed the efficacy of US-guided lavage with a corticosteroid injection compared with a corticosteroid injection in terms of calcification size reduction. A statistically significant change-from-baseline difference was observed in favour of the US-guided lavage group at 12 months, with a mean difference of $6.50 \mathrm{~mm}(95 \%$ CI 9.94 to $3.06, \mathrm{p}<0.01)$. No statistically significant difference was observed at 60 months between both interventions $(\mathrm{p}=0.86)$.

\section{Adverse effects, treatment cross-over and lost to follow-up results from all included studies}

No serious adverse events were reported in all three RCTs. In the RCT published by de Boer et $a l^{18} 1$ of the $11(9 \%)$ participants in the US-guided lavage decided to change treatment due to consistent pain whereas five participants $(36 \%)$ from the shockwave group decided to change treatment due to consistent pain. After 6 weeks, the Data Safety Monitoring Board decided to stop further enrolment in the study because of reported higher pain in the shockwave group. In the RCT published by Del Castillo-Gonzalez et al, ${ }^{20}$ no patient reported complications other than a slight discomfort due to the ESWT. However, 38 participants (31\%) from the shockwave group did not complete the intended treatment. Three participants in 


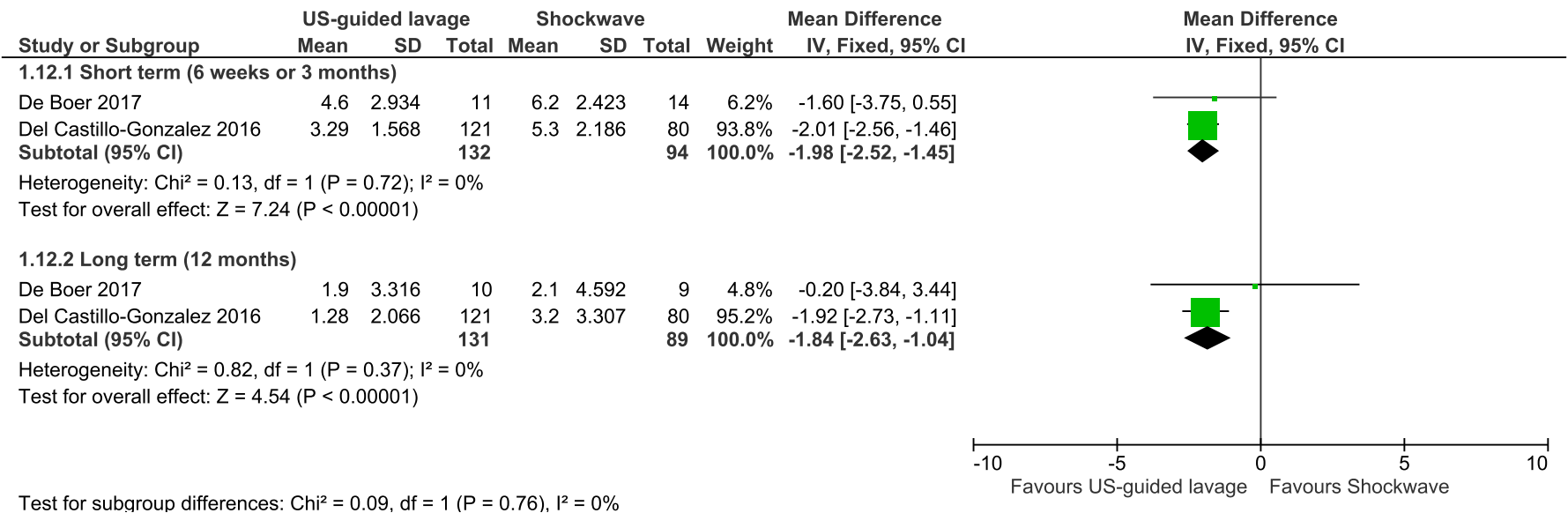

Figure 4 Forest plots of pooled studies ultrasound-guided lavage with a corticosteroid injection to shockwave therapy for change in pain in patient with chronic rotator cuff calcific tendinopathy. The squares are mean differences and the diamonds are pooled mean difference with $95 \%$ Cls. UGL, ultrasound-guided lavage.

the ESWT group did not attend a follow-up appointment compared with one in the US-guided lavage. These two studies did not analyse results according to the intentionto-treat principle. In the RCT published by de Witte $e t$ $a l,{ }^{16}{ }^{19}$ two participants developed a frozen shoulder in the US-guided lavage. Four of the 23 participants in the US-guided lavage underwent either a second US-guided lavage $(n=3)$ or a surgical intervention $(n=1)$ while 16 of the 25 participants in the corticosteroid injection group underwent either a US-guided lavage $(n=13)$ or a surgical intervention $(n=3)$.

\section{DISCUSSION}

The aim of this systematic review was to assess the efficacy of US-guided lavage for RC calcific tendinopathy. Three RCTs were included; one RCT was considered at low risk of bias and the two other trials were considered at high risk of bias.

Based on our meta-analyses of two RCTs with high risk of bias, there is low-quality evidence that US-guided lavage with a corticosteroid injection is more effective than shockwave therapy to reduce pain. The magnitude of the effect is probably clinically important in the short term since the mean difference is above the MCID. However, in the long term, it may or may not be clinically important since the MCID is comprised within the measured CI. These results need to be interpreted with caution since in both studies, the pain outcome was not clearly defined, and only per-protocol analyses were reported which may modify the magnitude of the observed effect.

Based on one high risk of bias RCT, ${ }^{18}$ there is low-quality evidence that US-guided lavage with a corticosteroid injection is more effective than shockwave therapy to improve function at 6 weeks based on the CMS. The magnitude of the difference observed may or may not be considered clinically important since it is within the range of previously reported MCID. However, no statistical difference between the two groups was observed on the Oxford Shoulder Score at 6 weeks and
12 months. Again, these results need to be interpreted with caution and more high-quality trials are needed to formally conclude on the superiority of US-guided lavage compared with shockwave therapy.

Based on one high risk of bias RCT, ${ }^{20}$ US-guided lavage with a corticosteroid injection is more effective than shockwave to reduce calcification size at different time points. Although previous epidemiological studies observed that the presence of a calcific deposit correlates with shoulder pain especially if the deposit is large, ${ }^{42627}$ it remains unclear if a reduction in the calcification size leads to pain reduction or to a functional improvement which are the most relevant outcomes to focus on when determining if US-guided lavage is efficacious to treat patients with RC calcific tendinopathy. Then, calcification size may be relevant to look at in a trial but only as a secondary outcome.

While our conclusions, based on low-quality evidence, state that US-guided lavage is to be preferred over shockwave therapy, previous meta-analyses for shockwave therapy conclude that it is superior to a placebo to improve function and to decrease the calcification size in individuals with chronic RC calcific tendinopathy. ${ }^{6}$ Then, it may still be clinically relevant to offer to patients a less invasive intervention such as shockwave therapy. Therefore, it is recommended to consider shockwave therapy and US-guided lavage when the initial conservative treatment fails, while arthroscopic surgery should only be considered as a last option. ${ }^{15}$

Based on one low risk of bias RCT, ${ }^{16} 19$ the addition of US-guided lavage to a corticosteroid injection appears to be more beneficial to improve function. The magnitude of the difference could be considered clinically important. Yet the measured effect was not consistently observed for all functional outcome measures and therefore more methodologically sound trials are warranted to formally conclude regarding US-guided lavage efficacy.

In regard to pain outcome, our results strengthen the conclusions of the reviews of Arirachakaran $e t \mathrm{al}^{14}$ and Wu 
et al. ${ }^{10}$ However, our results related to function contrast with those from Arirachakaran et al. ${ }^{14}$ It is important to highlight that Arirachakaran et $a l^{14}$ performed a network meta-analysis including only one RCT on US-guided lavage. ${ }^{16}$ They performed a direct comparison between US-guided lavage and a corticosteroid injection and indirect comparisons with a placebo and to US-guided needling fragmentation with shockwave which needs to be interpreted cautiously. ${ }^{17}$ We also excluded two RCTs which had been previously included in other systematic review because the intervention consisted of US-guided needling fragmentation and not US-guided lavage. ${ }^{28} 29$ In the RCT published by Kim $e t a l,{ }^{28}$ US-guided needling was found to be statistically more effective than extracorporeal shockwave therapy to improve function and to decrease pain at 1 year but not at 23 months. Moreover, US-guided needling was more effective to decrease calcification size at 23 months. In the other RCT on US-guided needling, Krasny et $a t^{29}$ observed that the addition of US-guided lavage to ESWT further improved function at a mean 4.1 months. There was no between group statistical difference in calcification size; still the rate of complete disappearance of calcium deposit was statistically higher in the US-guided needling group at a mean 4.1 months. It would be interesting to compare US-guided needling with US-guided lavage on pain and on function in a future RCT.

It is also important to highlight that the population in this systematic review was composed of patient with chronic RC calcific tendinopathy with symptoms for at least 3 months. The results from this systematic review with meta-analyses might not translate into acute or subacute RC calcific tendinopathy.

The diagnostic criteria in the included studies are also debatable. The calcification size is likely an important diagnostic criteria since larger RC calcifications tend to be more often symptomatic. ${ }^{4}{ }^{27}$ However, de Witte et $a l^{1619}$ used only $3 \mathrm{~mm}$ as a minimal calcification size while Del-Castillo Gonzalez et $a l^{20}$ used $5 \mathrm{~mm}$. De Boer et $a l^{18}$ did not include a minimal calcification size in the diagnostic criteria. Positive power Doppler signal within the RC calcific deposit has been associated with pain. ${ }^{27}$ However, none of the three RCTs used Doppler signal as a diagnostic criteria. Then, we can hypothesise that some participants in these three RCTs were not symptomatic because of the calcification deposit. The position of the calcification size on the tendon could also be of diagnostic value since a calcification in the middle of the tendon or at the articular surface could be less symptomatic than a calcification on the bursal surface of the tendon. Future studies may want to use Doppler signal as a diagnostic criteria and stratify their results based on the calcification size and the position of the calcification deposit on the tendon.

Initial conservative care involving an exercise programme with or without the use of medications may be the treatment of choice for acute and subacute RC calcific tendinopathy since it is less invasive than
US-guided lavage or a corticosteroid injection. However, this statement is not based on any RCT and further research should confirm what is the best initial conservative care for acute and subacute RC calcific tendinopathy.

No serious adverse effect was reported in the included trials. However, the small sample sizes are an important limit to this analysis since serious adverse effects such as infection are rare, and trials were likely underpowered to detect these differences between treatments. Although it is reported in the literature from systematic reviews on observational studies that US-guided lavage is a safe technique,${ }^{30}$ it remains to be fully answered in adequately powered RCTs. More pain has been reported with shockwave treatment, which needs to be considered when choosing this modality.

\section{Strengths and limitations}

Strengths of this review include the use of four important databases, a thorough search strategy and the use of the validated Cochrane risk-of-bias tool. However, this review also has limitations. The small sample size and the high risk of bias of two of the three articles are two important limitations of this review which limits the strength of our conclusions. Our meta-analysis only includes two RCTs, with the analysis heavily based on one RCT. The absence of comparison with a conservative treatment involving physical therapy with an exercise programme is another limitation of this systematic review.

\section{CONCLUSION}

In individuals with chronic RC calcific tendinopathy, low-quality evidence suggests that US-guided lavage with a corticosteroid injection is more effective than shockwave therapy to reduce pain in the short and long term and to improve function in the short term and that these effects may or may not be clinically important. Low-quality evidence suggests that the addition of US-guided lavage to a corticosteroid injection is beneficial to improve function at 12-month follow-up and that this effect may or may not be clinically important. Further studies could modify the present conclusions.

\section{Author affiliations}

${ }^{1}$ Maisonneuve-Rosemont Hospital Research Center, University of Montreal Affiliated Research Center, Montreal, Canada

${ }^{2}$ Department of Medicine, Faculty of Medicine, University of Montreal, Montreal, Canada

${ }^{3}$ University of Montreal Hospital Center Research Center (CRCHUM), Montreal, Canada

${ }^{4}$ Department of Radiology, Oncology and Nuclear Medicine, Faculty of Medicine, University of Montreal, Montreal, Canada

${ }^{5}$ School of Rehabilitation, Faculty of Medicine, University of Montreal, Montreal, Canada

${ }^{6}$ Department of Rehabilitation, Faculty of Medicine, Laval University, Quebec, Canada

${ }^{7}$ Center for Interdisciplinary Research in Rehabilitation and Social Integration (CIRRIS), Quebec, Canada

Contributors SL: designed the study protocol, performed the literature search, selected the articles of interest, performed the risk-of-bias analysis, extracted and analysed the data, and is the first author of the article. PD-C: assisted SL in the 
literature search and in the data analysis and reviewed the article. MS: performed the study selection and the risk-of-bias analysis with SL and reviewed the article. $\mathrm{ML}$ : assisted SL in the selection of the article especially regarding the ultrasoundguided lavage technique, assisted SL in the data analysis and reviewed the article. NJB: assisted SL in the selection of the article especially regarding the ultrasoundguided lavage technique, assisted SL in the data analysis and reviewed the article. J-OD: assisted SL to design the protocol and reviewed the article. J-SR: assisted SL to design the protocol and reviewed the article. FD: assisted SL in each step, managed the project and is the corresponding author.

Funding This article is part of a project supported by the Institut de recherche Robert-Sauvé en santé et sécurité au travail (IRSST). The grant number is 20160029. The project took place at Maisonneuve-Rosemont Hospital Research Center, University of Montreal Affiliated Research Center in Montreal, Quebec, Canada.

Competing interests All authors except NJB report grants from Institut de recherche Robert-Sauvé en santé et sécurité au travail (IRSST) during the conduct of the study which may be considered as a potential conflict of interest. However, since the IRSST is not a private company and does not have any financial interest in the treatment of shoulder disorders, this grant should not be considered as a conflict of interest. We still decided to declare this grant as a potential conflict of interest.

\section{Patient consent for publication Not required.}

Provenance and peer review Not commissioned; internally peer reviewed.

Open access This is an open access article distributed in accordance with the Creative Commons Attribution Non Commercial (CC BY-NC 4.0) license, which permits others to distribute, remix, adapt, build upon this work non-commercially, and license their derivative works on different terms, provided the original work is properly cited, appropriate credit is given, any changes made indicated, and the use is non-commercial. See: http://creativecommons.org/licenses/by-nc/4.0/.

\section{REFERENCES}

1. Greving K, Dorrestijn O, Winters JC, et al. Incidence, prevalence, and consultation rates of shoulder complaints in general practice. Scand $J$ Rheumatol 2012;41:150-5.

2. Tekavec E, Jöud A, Rittner R, et al. Population-based consultation patterns in patients with shoulder pain diagnoses. $B M C$ Musculoskelet Disord 2012;13.

3. Luime JJ, Koes BW, Hendriksen IJM, et al. Prevalence and incidence of shoulder pain in the general population; a systematic review. Scand J Rheumatol 2004;33:73-81.

4. Louwerens JKG, Sierevelt IN, van Hove RP, et al. Prevalence of calcific deposits within the rotator cuff tendons in adults with and without subacromial pain syndrome: clinical and radiologic analysis of 1219 patients. J Shoulder Elbow Surg 2015;24:1588-93.

5. Lippmann RK. Observations concerning the calcific cuff deposit. Clin Orthop 1961;20:49-60.

6. Louwerens JKG, Sierevelt IN, van Noort A, et al. Evidence for minimally invasive therapies in the management of chronic calcific tendinopathy of the rotator cuff: a systematic review and metaanalysis. J Shoulder Elbow Surg 2014;23:1240-9.

7. Gärtner J, Simons B. Analysis of calcific deposits in calcifying tendinitis. Clin Orthop Relat Res 1990;254:111-20.

8. Bureau N. Calcific tendinopathy of the shoulder. Semin Musculoskelet Radiol 2013;17:080-4.

9. Bosworth BM. Calcium deposits in the shoulder and subacromial bursitis: a survey of 12,122 shoulders. J Am Med Assoc 1941;116:2477-82.

10. Wu Y-C, Tsai W-C, Tu Y-K, et al. Comparative effectiveness of nonoperative treatments for chronic calcific tendinitis of the shoulder: a systematic review and network meta-analysis of randomized controlled trials. Arch Phys Med Rehabil 2017;98.

11. Gosens T, Hofstee D-J. Calcifying tendinitis of the shoulder: advances in imaging and management. Curr Rheumatol Rep 2009;11:129-34

12. Page MJ, Green S, Mrocki MA, et al. Electrotherapy modalities for rotator cuff disease. Cochrane Database Syst Rev 2016;6.
13. Vignesh KN, McDowall A, Simunovic N, et al. Efficacy of ultrasoundguided percutaneous needle treatment of calcific tendinitis. AJR Am J Roentgenol 2015;204:148-52.

14. Arirachakaran A, Boonard M, Yamaphai S, et al. Extracorporeal shock wave therapy, ultrasound-guided percutaneous lavage, corticosteroid injection and combined treatment for the treatment of rotator cuff calcific tendinopathy: a network meta-analysis of RCTs. Eur J Orthop Surg Traumatol 2017;27:381-90.

15. Louwerens JKG, Veltman ES, van Noort A, et al. The effectiveness of high-energy extracorporeal shockwave therapy versus ultrasoundguided needling versus arthroscopic surgery in the management of chronic calcific rotator cuff tendinopathy: a systematic review. Arthroscopy 2016;32:165-75.

16. de Witte PB, Selten JW, Navas A, et al. Calcific tendinitis of the rotator cuff: a randomized controlled trial of ultrasound-guided needling and lavage versus subacromial corticosteroids. Am J Sports Med 2013;41:1665-73.

17. Higgins JPT SG. Cochrane Handbook for Systematic Reviews of Interventions. The Cochrane Collaboration, 2011.

18. De Boer FA, Mocking F, Nelissen EM, et al. Ultrasound guided needling vs radial shockwave therapy in calcific tendinitis of the shoulder: a prospective randomized trial. J Orthop 2017;14:466-9.

19. de Witte PB, Kolk A, Overes F, et al. Rotator cuff calcific tendinitis: ultrasound-guided Needling and lavage versus subacromial corticosteroids: five-year outcomes of a randomized controlled trial. Am J Sports Med 2017;45:3305-14.

20. Del Castillo-González F, Ramos-Alvarez JJ, Rodríguez-Fabián $\mathrm{G}$, et al. Extracorporeal shockwaves versus ultrasound-guided percutaneous lavage for the treatment of rotator cuff calcific tendinopathy: a randomized controlled trial. Eur J Phys Rehabil Med 2016;52:145-51.

21. Tashjian RZ, Deloach J, Porucznik CA, et al. Minimal clinically important differences (MCID) and patient acceptable symptomatic state (PASS) for visual analog scales (VAS) measuring pain in patients treated for rotator cuff disease. J Shoulder Elbow Surg 2009;18:927-32.

22. Kukkonen J, Kauko T, Vahlberg T, et al. Investigating minimal clinically important difference for constant score in patients undergoing rotator cuff surgery. J Shoulder Elbow Surg 2013;22:1650-5

23. Holmgren T, Oberg B, Adolfsson L, et al. Minimal important changes in the Constant-Murley score in patients with subacromial pain. $J$ Shoulder Elbow Surg 2014;23:1083-90.

24. Ekeberg OM, Bautz-Holter E, Keller A, et al. A questionnaire found disease-specific WORC index is not more responsive than SPADI and OSS in rotator cuff disease. J Clin Epidemiol 2010;63:575-84.

25. Braun $\mathrm{C}$, Handoll $\mathrm{HH}$. Estimating the minimal important difference for the Western Ontario rotator cuff index (WORC) in adults with shoulder pain associated with partial-thickness rotator cuff tears. Musculoskelet Sci Pract 2018;35:30-3.

26. Sansone V, Consonni O, Maiorano E, et al. Calcific tendinopathy of the rotator cuff: the correlation between pain and imaging features in symptomatic and asymptomatic female shoulders. Skeletal Radiol 2016;45:49-55

27. Le Goff B, Berthelot J-M, Guillot P, et al. Assessment of calcific tendonitis of rotator cuff by ultrasonography: comparison between symptomatic and asymptomatic shoulders. Joint Bone Spine 2010;77:258-63.

28. Kim Y-S, Lee H-J, Kim Y-vin, et al. Which method is more effective in treatment of calcific tendinitis in the shoulder? Prospective randomized comparison between ultrasound-guided needling and extracorporeal shock wave therapy. J Shoulder Elbow Surg 2014;23:1640-6.

29. Krasny C, Enenkel M, Aigner N, et al. Ultrasound-guided needling combined with shock-wave therapy for the treatment of calcifying tendonitis of the shoulder. J Bone Joint Surg Br 2005;87:501-7.

30. Gatt DL, Charalambous CP. Ultrasound-guided barbotage for calcific tendonitis of the shoulder: a systematic review including 908 patients. Arthroscopy 2014;30:1166-72.

31. Lanza E, Banfi G, Serafini G, et al. Ultrasound-guided percutaneous irrigation in rotator cuff calcific tendinopathy: what is the evidence? A systematic review with proposals for future reporting. Eur Radiol 2015;25:2176-83. 